\title{
Hemodynamic regulation of perivalvular endothelial gene expression prevents deep venous thrombosis
}

\author{
John D. Welsh, ${ }^{1}$ Mark H. Hoofnagle, ${ }^{2}$ Sharika Bamezai, ${ }^{1}$ Michael Oxendine, ${ }^{3}$ Lillian Lim, ${ }^{1}$ Joshua D. Hall, ${ }^{4}$ Jisheng Yang, ${ }^{1}$ \\ Susan Schultz, ${ }^{5}$ James Douglas Engel, ${ }^{6}$ Tsutomu Kume, ${ }^{3}$ Guillermo Oliver, ${ }^{3}$ Juan M. Jimenez, ${ }^{4}$ and Mark L. Kahn ${ }^{1}$ \\ 'Department of Medicine and Cardiovascular Institute, University of Pennsylvania, Philadelphia, Pennsylvania, USA. ²Department of Surgery, Division of Traumatology, Surgical Critical Care, and Emergency \\ Surgery, Hospital of the University of Pennsylvania, Philadelphia, Pennsylvania, USA. ${ }^{3}$ Center for Vascular and Developmental Biology, Feinberg Cardiovascular Research Institute, Northwestern University, \\ Chicago, Illinois, USA. ${ }^{4}$ Department of Mechanical and Industrial Engineering, University of Massachusetts Amherst, Amherst, Massachusetts, USA. ${ }^{5}$ Department of Radiology, Hospital of the University of \\ Pennsylvania, Philadelphia, Pennsylvania, USA. ${ }^{6}$ Department of Cell and Developmental Biology, University of Michigan, Ann Arbor, Michigan, USA.
}

\begin{abstract}
Deep venous thrombosis (DVT) and secondary pulmonary embolism cause approximately 100,000 deaths per year in the United States. Physical immobility is the most significant risk factor for DVT, but a molecular and cellular basis for this link has not been defined. We found that the endothelial cells surrounding the venous valve, where DVTs originate, express high levels of FOXC2 and PROX1, transcription factors known to be activated by oscillatory shear stress. The perivalvular venous endothelial cells exhibited a powerful antithrombotic phenotype characterized by low levels of the prothrombotic proteins vWF, P-selectin, and ICAM1 and high levels of the antithrombotic proteins thrombomodulin (THBD), endothelial protein C receptor (EPCR), and tissue factor pathway inhibitor (TFPI). The perivalvular antithrombotic phenotype was lost following genetic deletion of FOXC2 or femoral artery ligation to reduce venous flow in mice, and at the site of origin of human DVT associated with fatal pulmonary embolism. Oscillatory blood flow was detected at perivalvular sites in human veins following muscular activity, but not in the immobile state or after activation of an intermittent compression device designed to prevent DVT. These findings support a mechanism of DVT pathogenesis in which loss of muscular activity results in loss of oscillatory shear-dependent transcriptional and antithrombotic phenotypes in perivalvular venous endothelial cells, and suggest that prevention of DVT and pulmonary embolism may be improved by mechanical devices specifically designed to restore perivalvular oscillatory flow.
\end{abstract}

\section{Introduction}

Deep venous thrombosis (DVT) is a common vascular disease with an annual incidence of $0.1 \%$ among the general population, and more than $1 \%$ among hospitalized individuals (1). Pulmonary embolism - the blockade of pulmonary flow caused by a DVT that becomes dislodged and travels through the venous system to the lungs - is the third most common cause of cardiovascular death after myocardial infarction and stroke (2). Unlike myocardial infarction and stroke, DVT is not a thrombotic complication of atherosclerosis, and present therapy is limited to systemic anticoagulation and intermittent compression devices (ICDs) designed to augment venous flow.

In 1856 Rudolph Virchow proposed a mechanistic triad for DVT pathogenesis that included stasis, defined as changes in venous blood flow associated with immobility; local vessel injury;

Conflict of interest: JDW and MLK are coinventors on a patent, PCTUS1932399, entitled "Medical device for the prevention of thrombosis" that is based on the scientific discoveries described in this article. They are stakeholders in a company, Osciflex, that has an option agreement with the University of Pennsylvania to develop a new device to prevent deep venous thrombosis.

Copyright: @ 2019, American Society for Clinical Investigation.

Submitted: September 10, 2018; Accepted: September 18, 2019;

Published: November 11, 2019

Reference information: J Clin Invest. 2019;129(12):5489-5500.

https://doi.org/10.1172/JCl124791. and hypercoagulability (3). The role of hypercoagulability in DVT pathogenesis has since been proven by the increased risk of DVT among individuals with gain-of-function mutations in clotting factors (e.g., factor V Leiden; refs. 4, 5), or activation of the clotting system by trauma, surgery, or circulating tissue factor-positive tumor microparticles (reviewed in ref. 6). In contrast, although epidemiologic studies have identified immobility as the strongest risk factor for DVT (1), neither a clear molecular basis for the role of stasis/immobility nor evidence of local vessel injury has been demonstrated in DVT pathogenesis.

An important clue regarding the pathogenesis of DVT was obtained over a half century ago when autopsy studies revealed that most DVTs originate in the sinus of venous valves, i.e., the space between the valve leaflet and the adjacent vessel wall (Figure $1 \mathrm{~A}$ and refs. 7-9). Venography revealed that circulating blood is retained longer in the valve sinus than the nonvalvular venous lumen, suggesting that this might predispose to clot formation at that site (10). These insights implicated the perivalvular region of the vein in DVT formation, but a clear mechanistic understanding of the relationship between venous valves and DVT pathogenesis has been lacking.

In the present study we demonstrate that the endothelial cells that line the venous valve sinus and adjacent valve leaflet (collectively referred to as the perivalvular endothelium) exhibit high expression of the FOXC2 and PROX1 transcription factors in asso- 
A

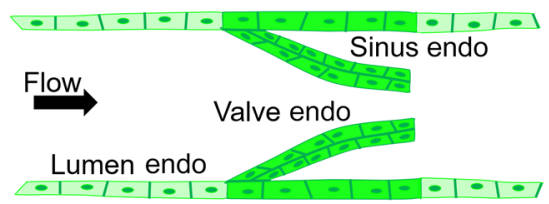

B

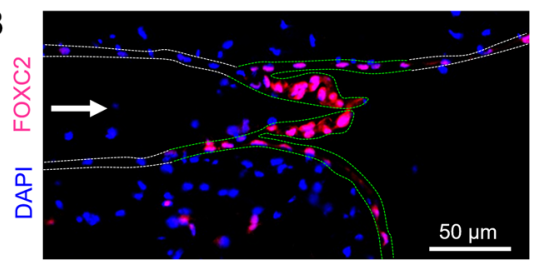

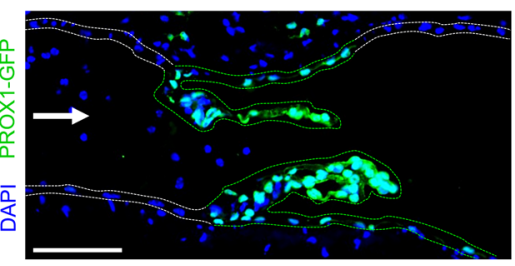

C
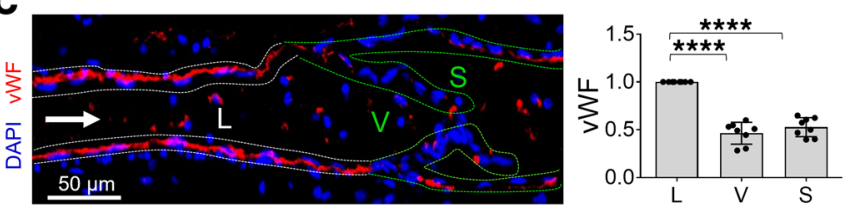

E
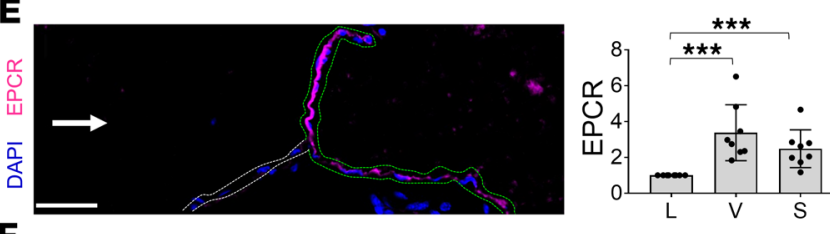

D
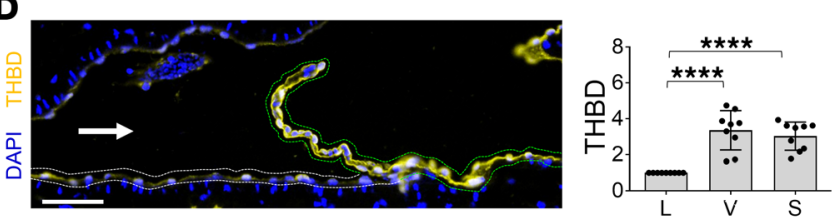

$\mathbf{F}$
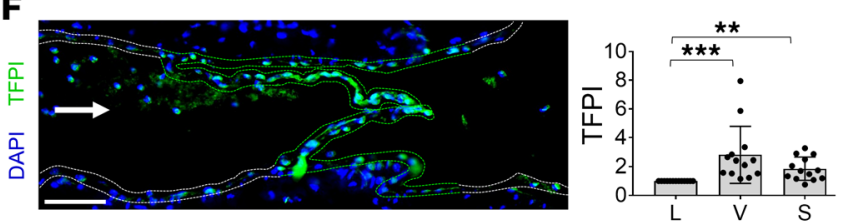

G
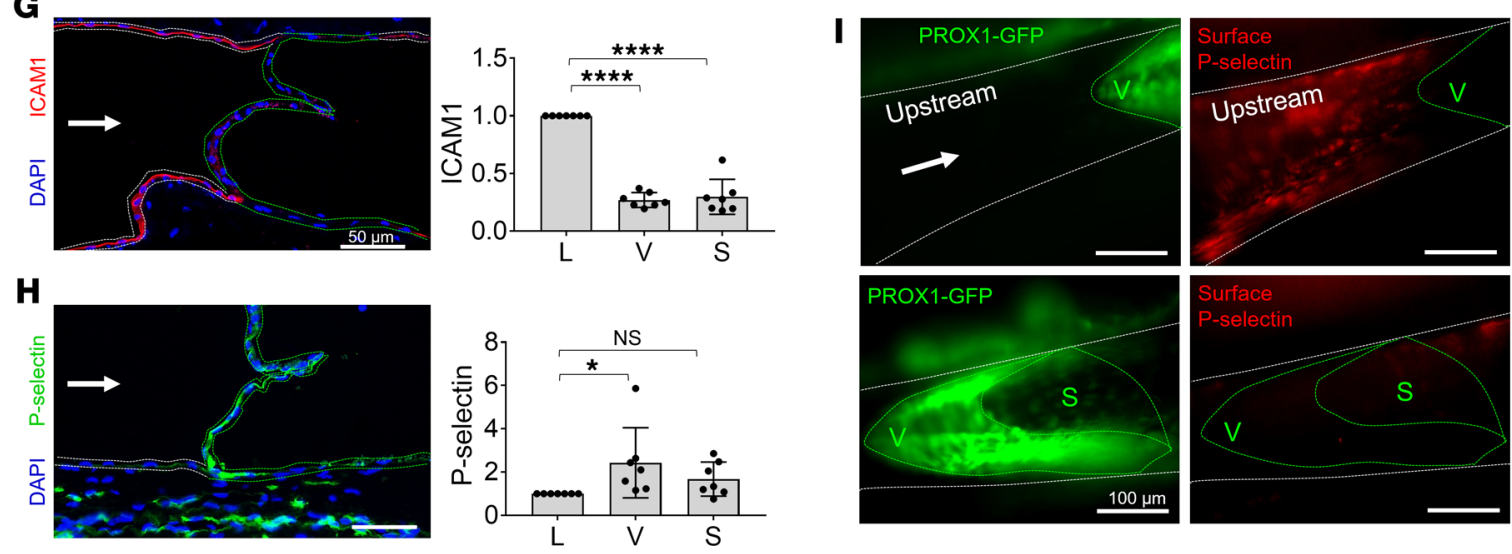

Figure 1. FOXC2- and PROX1-expressing endothelial cells around the venous valve exhibit a strong antithrombotic phenotype. (A) Schematic illustration of mouse venous valve sections defining the regions of luminal endothelium (Lumen endo), valve leaflet endothelium (Valve endo), and valve sinus endothelium (Sinus endo). (B) FOXC2 and PROX1 were measured using anti-FOXC2 and anti-GFP immunostaining of wild-type and PROX1-GFP transgenic mice. Cell nuclei were detected with DAPI staining. White dashed lines indicate luminal venous endothelial cells; green dashed lines indicate perivalvular endothelial cells. Arrows indicate the direction of venous blood flow. Images are representative of 8 separate experiments using different animals. (C-F) Mouse saphenous veins were immunostained to detect expression of vWF ( $n=8$ valves), THBD $(n=9)$, EPCR $(n=8)$, and TFPI $(n=13)$. Relative quantitation of staining in luminal (L), valvular (V), and sinus (S) endothelial cells is shown at right for each protein. (G and $\mathbf{H})$ Mouse saphenous veins were immunostained to detect expression of the adhesion proteins ICAM1 $(n=7)$ and P-selectin $(n=7)$. Relative quantitation of protein levels is shown at right for each protein. (I) P-selectin is not expressed on the surface of perivalvular endothelial cells. Surface P-selectin was detected by i.v. injection of Alexa Fluor 647-labeled anti-P-selectin antibodies into PROX1-GFP transgenic animals. Images are representative of 6 separate experiments in 4 mice. White dashed lines indicate luminal venous endothelial cells, and green dashed lines indicate perivalvular endothelial cells. Arrows indicate the direction of venous blood flow. For each graph the mean is shown as the bar with dots representing each data point, and error bars indicate SD. Significance was determined by ratio paired $t$ test and corrected for multiple comparisons. ${ }^{*} P<0.025 ;{ }^{* *} P<0.01 ;{ }^{* *} P<0.001$; ${ }^{* * * *} P<0.0001$.

ciation with a strong antithrombotic phenotype, characterized by low levels of the prothrombotic proteins von Willebrand factor (vWF), P-selectin, and intercellular adhesion molecule 1 (ICAM1) and high levels of the antithrombotic proteins thrombomodulin (THBD), endothelial protein $\mathrm{C}$ receptor (EPCR), and tissue factor pathway inhibitor (TFPI). Loss of this perivalvular antithrombotic, antiinflammatory endothelial phenotype is observed following loss of venous flow or genetic deletion of Foxc2 or Prox1 in mice. Loss of this unique, antithrombotic perivalvular endothelial phenotype is also observed at the site of origin of human DVT. Finally, Doppler ultrasound studies of human leg veins demonstrate that the perivalvular region experiences strong oscillatory blood flow following muscular activity that is absent in the immobile state and not reproduced by a clinically used ICD. These studies support a mechanism in which immobility results in DVT due to loss of a hemodynamically regulated antithrombotic endothelial cell phenotype at the venous valve, and suggest that new devices designed to specifically restore perivalvular oscillatory flow in the leg veins will more effectively prevent DVT and pulmonary embolism.

\section{Results}

Perivalvular venous endothelial cells express FOXC2 and PROX1 in association with a strong antithrombotic phenotype. Lymphatic valves develop in response to oscillatory shear stress (OSS) 
A
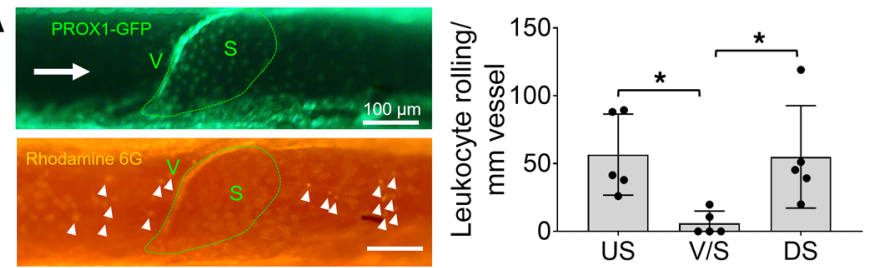

B
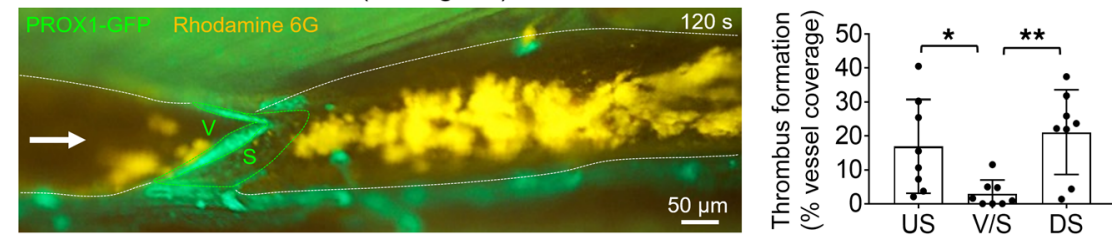

C
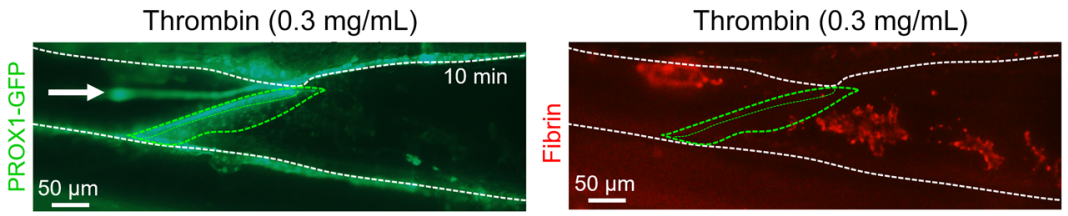

Figure 2. Perivalvular venous endothelium is resistant to leukocyte rolling and thrombus formation. (A) Spontaneous leukocyte rolling at the saphenous venous valve was observed and quantitated in PROX1-GFP mice using rhodamine 6C. Arrow indicates the direction of flow, and arrowheads indicate individual leukocytes adherent to the vessel wall. Dashed lines outline the valve sinus region. The number of rolling leukocytes per millimeter vessel length was measured upstream of the valve (US), in the valve and valve sinus regions (V/S), and downstream of the valve (DS) (right) ( $n=5$ valves imaged in 3 mice). (B) Thrombus formation was stimulated in PROX1-GFP animals around the saphenous venous valve by application of extravascular thrombin, and clot visualized by accumulation of rhodamine 6G-positive platelets and leukocytes (orange) at 120 seconds (left). The percentage of vessel area covered by thrombus was measured upstream of the valve (US), in the valve and valve sinus regions (V/S), and downstream of the valve (DS) (right) ( $n=8$ valves imaged in 4 mice). (C) Visualization of thrombus formation over the saphenous venous valve of a PROX1-GFP mouse using anti-fibrin antibodies 10 minutes after thrombin application. The images are representative of those obtained from analysis of 3 separate experiments. White dashed lines indicate luminal venous endothelial cells, and green dashed lines indicate perivalvular endothelial cells. For each graph the mean is shown as the value of the bar with dots representing each replicate, and error bars indicate SD. Significance was determined by a paired 2-tailed $t$ test and corrected for multiple comparisons. ${ }^{*} P<0.025 ;{ }^{*} P<0.01$

through upregulation of the FOXC2, GATA2, and PROX1 transcription factors in perivalvular endothelial cells (11-17), and sustained expression of FOXC2 and GATA2 is required to maintain lymphatic valves in the mature animal $(16,18)$. Since venous valves are morphologically identical to lymphatic valves and also require FOXC2 and PROX1 to develop (19-22), we assessed whether this transcriptional program might be maintained in the endothelium surrounding the mature venous valve. Immunostaining of mouse saphenous veins from wild-type animals and PROX1-GFP transgenic reporter animals revealed that FOXC2 and PROX1 were specifically expressed in endothelial cells lining both sides of the venous valve and the adjacent valve sinus, but were undetectable in nonvalvular, lumenal venous endothelium (Figure 1, A and B). Analysis of transgenic GATA2-GFP reporter animals also revealed specific expression in perivalvular venous endothelial cells (Supplemental Figure 1A; supplemental material available online with this article; https://doi.org/10.1172/JCI124791DS1), but immunostaining with anti-GATA2 antibodies failed to detect a significant difference between luminal and perivalvular endothelial cells
(Supplemental Figure 1B). To determine whether this perivalvular expression pattern is conserved across species, we examined expression of these transcription factors in healthy human saphenous veins harvested for vascular bypass surgery. As observed in the mouse, FOXC2 and PROX1 were detected in the nuclei of endothelial cells lining the sinus (S) and downstream/sinus side of the human saphenous venous valve leaflet $\left(\mathrm{V}^{\mathrm{s}}\right)$, but not in nonvalvular lumenal venous endothelial cells (L) (Supplemental Figure $2 \mathrm{~A})$. In contrast to the mouse, the endothelial cells lining the upstream/lumenal side of the human venous valve leaflet $\left(\mathrm{V}^{\mathrm{L}}\right)$ did not express FOXC2 or PROX1 (Supplemental Figure 2A). As observed using anti-GATA2 antibodies in the mouse, immunodetection of GATA2 in human venous endothelium was not restricted to perivalvular endothelial cells (Supplemental Figure 1C). The differences between human and mouse FOXC2 and PROX1 endothelial expression on the luminal side of the venous valve leaflet may reflect hemodynamic differences, due either to differences in venous flow (e.g., in an upright, 2-legged human versus a horizontal, 4-legged rodent) or differences in vessel and valve size. Since these studies did not definitively establish a perivalvular expression pattern for native GATA2, we focused subsequent studies on FOXC2 and PROX1, which are highly and specifically expressed by perivalvular venous endothelial cells in both mice and humans.

Human perivalvular venous endothelial cells express low levels of the endothelial cell-associated prothrombotic protein vWF and high levels of the endothelial cell-associated antithrombotic proteins THBD and EPCR (23), suggesting that OSS and FOXC2 and PROX1 expression might be connected to regulation of the endothelial thrombotic phenotype at venous valves. Immunostaining of mouse saphenous veins revealed dramatic loss of vWF expression and gain of THBD, EPCR, and TFPI expression in perivalvular endothelial cells compared with nonvalvular, lumenal endothelial cells (Figure 1, C-F). Since venous thrombi incorporate adhesive leukocytes in addition to platelets and cross-linked fibrin $(8,24-26)$, we next assessed endothelial expression of the prothrombotic leukocyte adhesion proteins P-selectin and ICAM1. As observed for the prothrombotic protein vWF, ICAM1 expression was markedly downregulated in the perivalvular venous endothelium (Figure 1G). P-selectin expression appeared to be maintained or even increased when examined by immunostaining of tissue sections (Figure 1H), but intravascular injection of anti-P-selectin antibodies revealed dramatic loss of surface P-selectin in live perivalvular endothelial cells (Figure 1I). The observed discrepancy between intracellular and surface P-selectin expression is consis- 


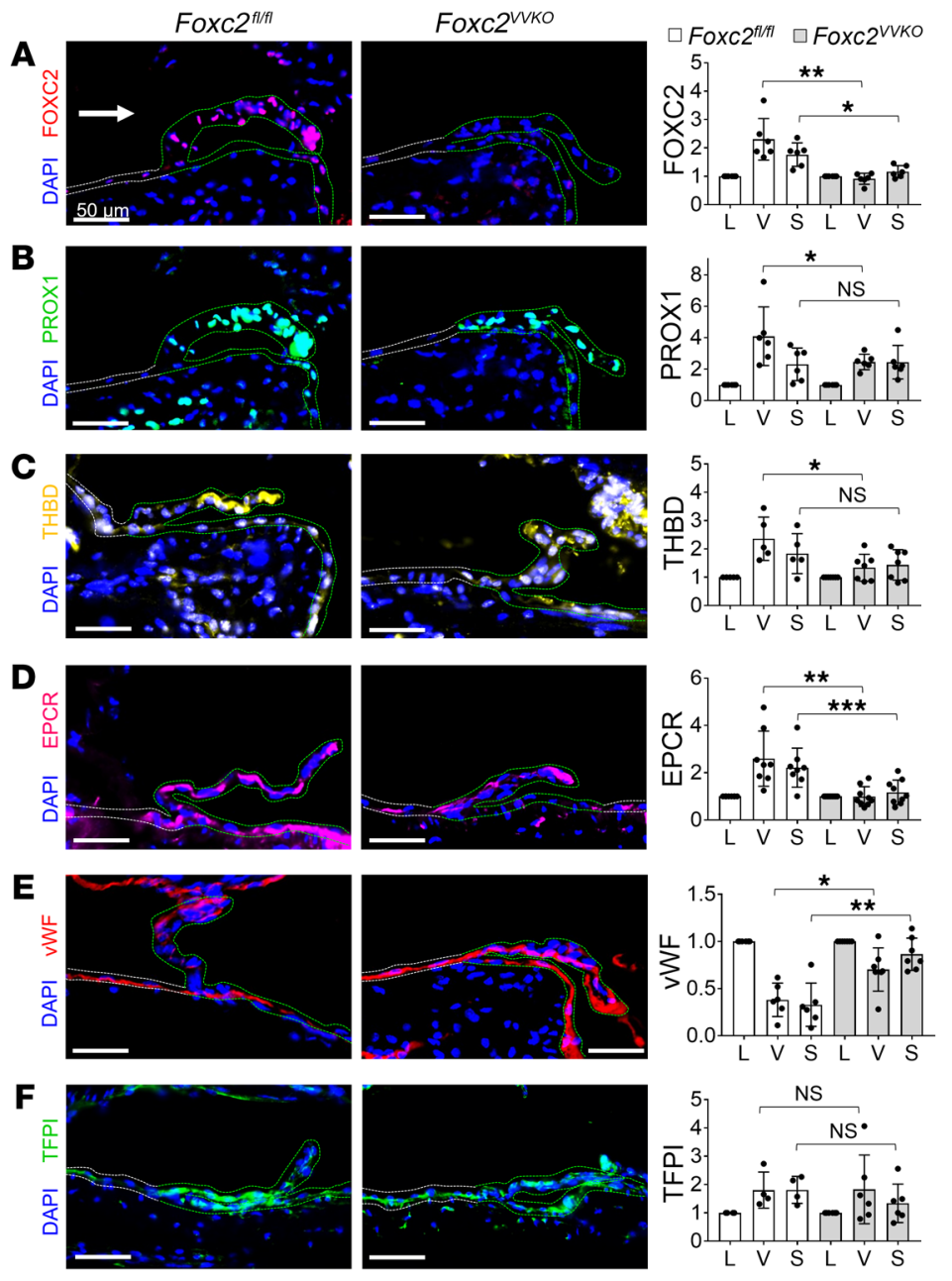

Figure 3. Loss of FOXC2 results in loss of THBD and EPCR and gain of $\mathbf{W W}$ in perivalvular venous endothelial cells. Immunostaining of mouse saphenous veins was performed in animals lacking FOXC2 in perivalvular venous endothelial cells ( $F o x c 2^{v v K O}$ mice) and littermate

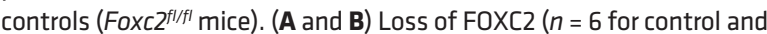
knockout valves) mildly reduces perivalvular endothelial expression of PROX1 ( $n=6$ for control and knockout valves). (C-E) Loss of FOXC2 results in loss of perivalvular endothelial expression of the antithrombotic proteins THBD ( $n=5$ control valves, $n=7$ knockout valves) and EPCR ( $n=8$ control valves, $n=9$ knockout valves) and gain of the prothrombotic protein vWF ( $n=6$ control valves, $n=7$ knockout valves). (F) Loss of FOXC2 does not significantly alter the expression of the antithrombotic protein TFPI ( $n=4$ control valves, $n=6$ knockout valves). White dashed lines indicate luminal venous endothelial cells, and green dashed lines indicate perivalvular endothelial cells. For all graphs the mean is represented by the bar with dots for each replicate, and error bars indicate SD. Significance for each single comparison was determined by an unpaired 2-tailed Mann-Whitney test. ${ }^{*} P<0.05$; ${ }^{* *} P<0.01 ;{ }^{* * *} P<0.005$. Scale bars: $50 \mu \mathrm{m}$. tent with studies demonstrating a requirement for vWF for surface expression of P-selectin, and suggests that valve sinus endothelium lacks functional Weibel-Palade bodies (27). Analysis of human saphenous veins also revealed marked upregulation of THBD, EPCR, and TFPI expression and downregulation of vWF expression in perivalvular endothelial cells (Supplemental Figure 2, B-E). Significantly, these changes were observed specifically in the endothelial cells lining the valve sinus (S) and downstream/sinus side of the valve leaflet $\left(\mathrm{V}^{\mathrm{S}}\right)$ in the human vein versus those lining the sinus and both sides of the valve leaflet in the mouse vein, a spatial pattern identical to that observed for expression of the shear-regulated transcription factors FOXC2 and PROX1 (Figure 1B and Supplemental Figure 2A). These studies reveal that the healthy perivalvular venous endothelium expresses a host of cell-associated proteins that are highly antithrombotic, and that this unique endothelial phenotype overlaps precisely with expression of the hemodynamically regulated FOXC2 and PROX1 transcription factors.

The perivalvular venous endothelium is highly antithrombotic. To functionally test whether the endothelial molecular phenotype described above confers a localized antithrombotic phenotype to the venous valve, we next observed leukocyte rolling in the saphenous vein of live PROX1-GFP mice, in which endothelial cells in the venous valve leaflets and sinus are marked by high GFP expression. Following injection of rhodamine $6 \mathrm{G}$, leukocyte roll- ing was observed both upstream and downstream of the venous valve and sinus, but no rolling leukocytes were observed on perivalvular endothelium (Figure 2A). To functionally test whether perivalvular venous endothelium is more or less thrombotic than adjacent nonvalvular venous endothelium, a low concentration of active thrombin $(0.3 \mathrm{mg} / \mathrm{mL})$ was applied to the exposed saphenous vein and thrombus formation detected using platelet and leukocyte uptake of rhodamine or anti-fibrin antibodies. Adherent thrombi were observed both upstream and downstream of the venous valve 2 minutes after thrombin application, but almost no clot formation was noted along the valve leaflets or in the valve sinus (Figure 2B). Consistent with these observations, robust fibrin formation was detected upstream and downstream of the PROX1-GFP ${ }^{+}$perivalvular region, but not at the valve itself, 10 minutes after thrombin application (Figure 2C). These findings demonstrate that the perivalvular venous endothelium is strongly antithrombotic in healthy mice.

FOXC2 and PROX1 confer the antithrombotic phenotype of perivalvular venous endothelial cells. The studies described above suggested that the transcription factors FOXC2 and PROX1 might couple hemodynamic forces to a strong antithrombotic phenotype in the endothelial cells that line the venous valve leaflets and sinus. To test the role of these transcription factors at the venous valve in vivo, we next deleted Foxc2 in the PROX1 ${ }^{+}$perivalvular 

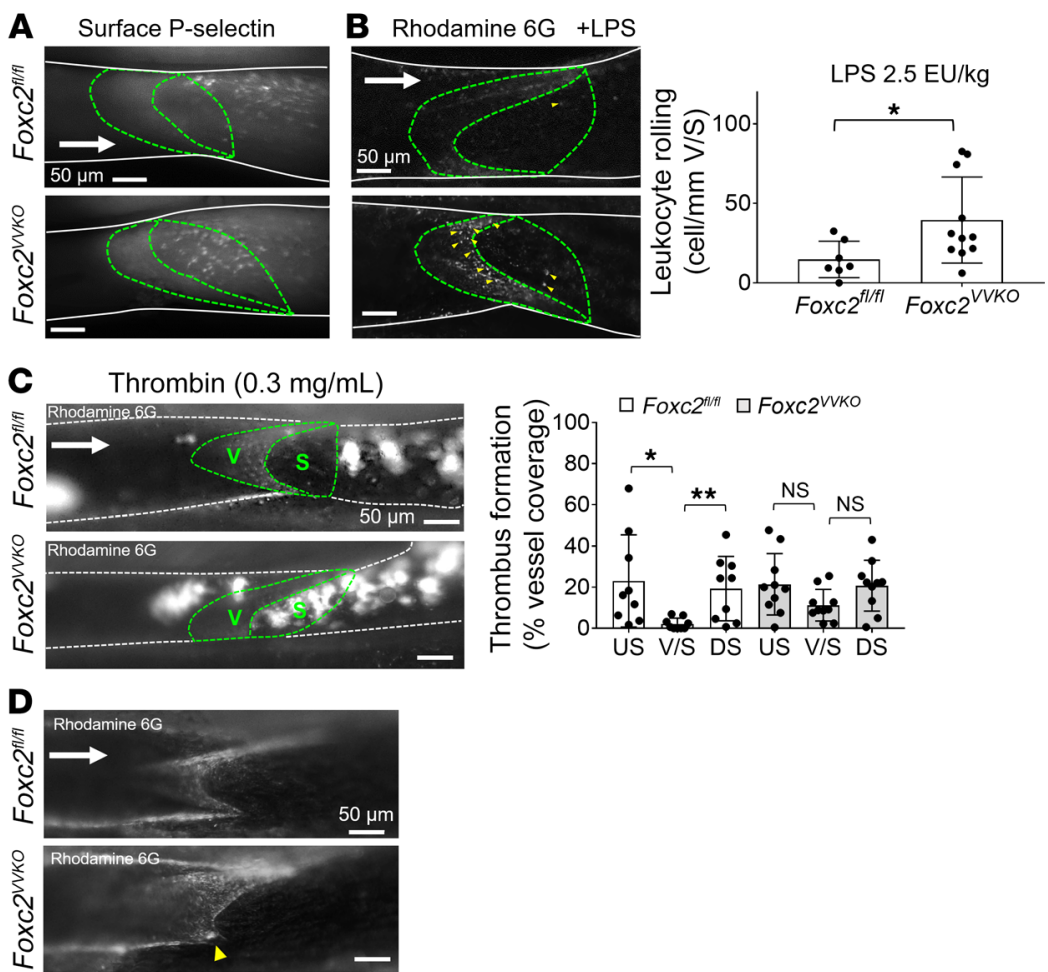

Figure 4. Loss of FOXC2 increases leukocyte rolling and thrombosis at the venous valve.

(A) Spontaneous leukocyte rolling at the saphenous venous valve was measured in Foxc2 ${ }^{\text {VVKo }}$ mice and littermate Foxc $2^{f / f f l}$ controls using rhodamine $6 \mathrm{G}$. Arrow indicates direction of flow; arrowheads indicate leukocytes adherent to the vessel wall. Dashed lines outline the valve sinus region. Images are representative of 6 similar experiments. (B) Leukocyte rolling after administration of the proinflammatory agent lipopolysaccharide (LPS) at the saphenous venous valve was measured Foxc ${ }^{V V K O}$ mice ( $n=7$ valves imaged in 4 mice) and littermate Foxc $2^{f / f l}$ controls ( $n=11$ valves imaged in 6 mice) using rhodamine $6 \mathrm{C}$. The mean is represented by the bar; each dot indicates a replicate. The error bars indicate SD. Significance was measured with an unpaired 2 -tailed $t$ test; ${ }^{*} P<0.05$. (C) Thrombus formation at the saphenous venous valve stimulated by application of extravascular thrombin was measured in animals lacking FOXC2 in perivalvular venous endothelial cells (Foxc2 ${ }^{V V K O}$ mice, $n=$ 9 valves imaged in 5 mice) and littermate controls (Foxc $2^{f / f l}$ mice, $n=10$ valves imaged in 5 mice) using rhodamine 6C (left). The percentage of vessel area covered by thrombus was measured upstream of the valve (US), in the valve and valve sinus regions (V/S), and downstream of the valve (DS) (right). The mean is represented by the bar; each dot indicates a replicate. Error bars indicate SD. Significance was determined by a paired 2 -tailed $t$ test and corrected for multiple comparisons. ${ }^{*} P<0.025 ;{ }^{*} P<0.01$. (D) The presence of perivalvular spontaneous microthrombi was measured in the indicated animals using rhodamine 6C (yellow arrowhead). Images are representative of 14 control valves and 17 knockout valves imaged; the quantitative data are found in Supplemental Table 1.

venous endothelial cells of $\operatorname{Prox} 1^{\text {CreRRT2 }}$ Fox $2^{\text {f/fll }}$ mature animals (termed Foxc2 $2^{\text {VVKO }}$ animals). Consistent with published studies (18), 3 weeks after tamoxifen-induced gene deletion Foxc2 ${ }^{\text {VKo }}$ animals appeared healthy, exhibited no loss of venous valves, and displayed no signs of edema, ascites, or lymphatic dysfunction. FOXC2 protein expression was not detectable in the perivalvular venous endothelial cells of Foxc2 ${ }^{\text {VVKO }}$ animals, while PROX1 expression was significantly reduced in the valve endothelium but remained elevated in comparison with lumenal endothelium (Figure 3, A and B). Significantly, perivalvular endothelial cells in Foxc2 ${ }^{\text {VVKO }}$ animals exhibited reduced expression of the antithrombotic proteins THBD and EPCR (Figure 3, C and D) and increased expression of the prothrombotic protein vWF (Figure 3E). Notably, perivalvular TFPI expression was not significantly altered in Foxc2 ${ }^{V V K O}$ animals (Figure 3F).

To assess the role of PROX1 in the perivalvular antithrombotic endothelial phenotype described above, we next analyzed saphenous venous valves from Cdh5-CreERT2 Prox $f^{\text {I/fl }}$ mature animals (termed Prox $1^{\text {ECKO }}$ animals). Prox $1^{\text {ECKO }}$ venous valves exhibited loss of endothelial PROX1 expression (Supplemental Figure 3A), but retained expression of FOXC2 in perivalvular endothelial cells (Supplemental Figure 3B). Unlike loss of FOXC2, loss of PROX1 did not significantly lower the expression of THBD or EPCR or raise the expression of $\mathrm{VWF}$ in perivalvular endothelial cells (Supplemental Figure 3, C-E). However, loss of PROX1 did significantly reduce perivalvular expression of TFPI (Supplemental Figure 3F), a protein that was not significantly altered with loss of FOXC2 (Figure 3F). Neither loss of FOXC2 nor loss of PROX1 altered ICAM1 expression (Supplemental Figure 4, A and B). These studies demonstrate that both FOXC2 and PROX1 are required for the antithrombotic phenotype of perivalvular venous endothelial cells and suggest that they function in discrete ways in this context.

Endothelial FOXC2 is required for protection against venous perivalvular thrombosis. Since Foxc2 ${ }^{\text {VVKO }}$ animals exhibited the most significant loss of the antithrombotic phenotype in perivalvular endothelial cells (Figure 3), we next asked whether loss of FOXC2 might predispose toward thrombosis at the venous valve in vivo. In contrast to Foxc $2^{f / f l}$ control littermates, Foxc2 ${ }^{\text {VVKO }}$ animals expressed P-selectin on the surface of perivalvular endothelial cells (Figure 4A). Foxc2 ${ }^{\text {VVKO }}$ animals also exhibited higher levels of leukocyte rolling over perivalvular venous endothelial cells than control littermates following injection of a low dose of lipopolysaccharide, a known activator of vascular endothelium (refs. 28, 29 , and Figure 4B). To examine the local thrombotic effects of FOXC2 deficiency in the perivalvular endothelium, we next tested protection against thrombin-induced clot formation. In contrast to control littermates, Foxc ${ }^{\text {VVKO }}$ animals exhibited clot formation both in the valve sinus and on the valve leaflets (Figure 4C). Significantly, live imaging immediately following exposure of the saphenous vein revealed spontaneous microthrombi along the valve leaflets and sinus in 6 of 17 Foxc2 ${ }^{\text {VVKO }}$ animals and 0 of 14 Foxc $2^{f / f l}$ controls (Figure 4D and Supplemental Table 1). No large changes in systemic clotting were observed using either the activated partial thromboplastin time (APTT) or fibrin clot time in Foxc2 ${ }^{\text {VVKO }}$ animals (Supplemental Figure 5). Pretreatment of the Foxc $2^{\text {VVKO }}$ and Foxc $2^{A / / l}$ animals with low-dose lipopolysaccharide 24 hours before valve exposure to increase the systemic hypercoagulability of the animals increased the rate of spontaneous thrombus formation to $8 / 12$ for the $\mathrm{Foxc}^{\text {VVKO }}{ }^{\text {Vnimals }}$ but only $1 / 8$ for the $\operatorname{Foxc}^{f / f l}$ animals (Supplemental Figure 6 and Supplemental Table 1). Notably, spontaneous thrombi in lipopoly- 

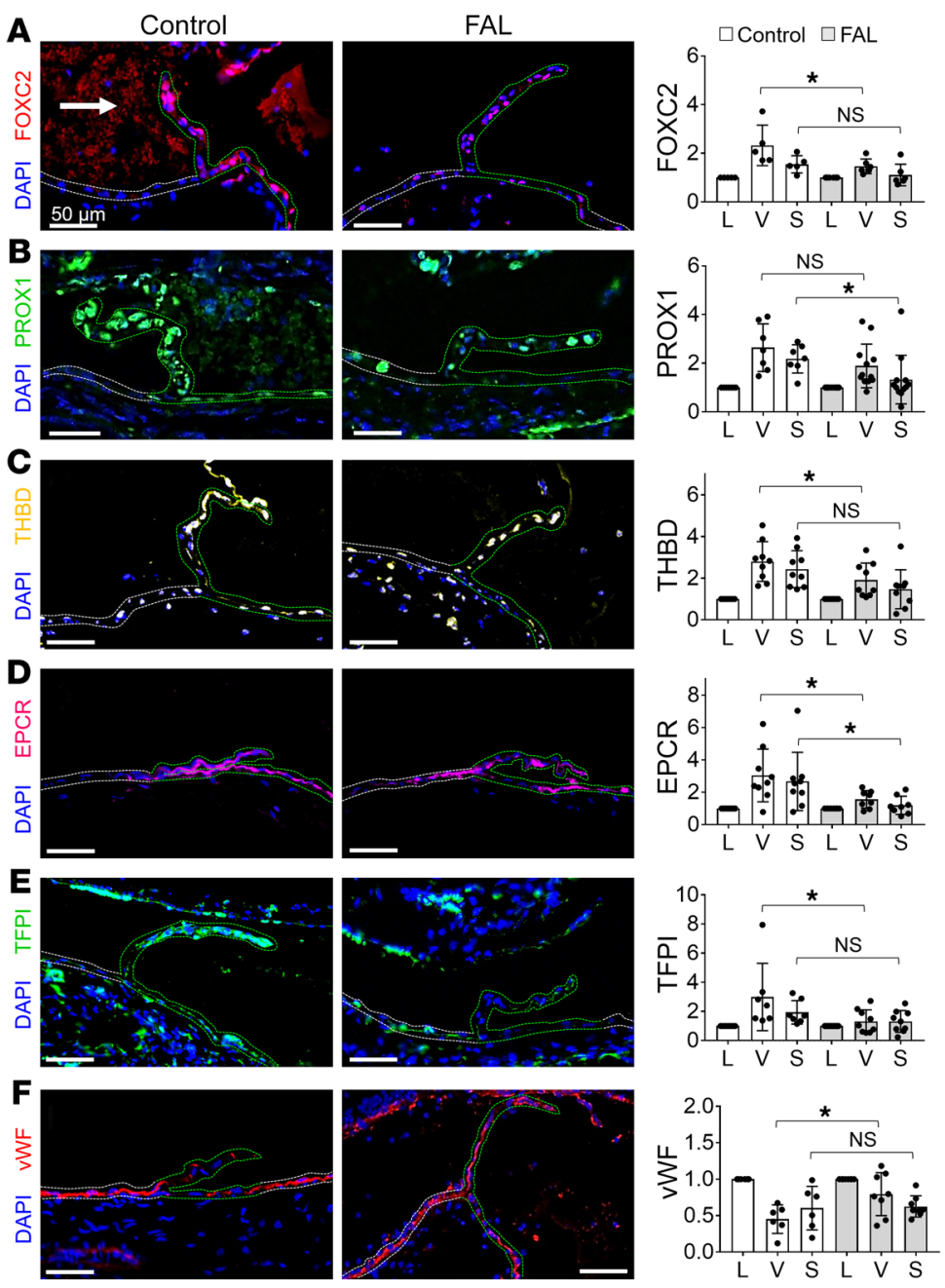

Figure 5. Femoral artery ligation results in loss of the venous perivalvular transcriptional and antithrombotic phenotypes. Immunostaining of mouse saphenous veins was performed 72 hours after femoral artery ligation (FAL) to reduce venous flow, and relative quantitation of protein levels in luminal $(L)$, valvular $(V)$, and sinus (S) endothelial cells was measured. (A and B) Loss of perivalvular endothelial expression of the FOXC2 ( $n=5$ control, $n=6$ FAL valves) and PROX1 ( $n=7$ control, $n=12$ FAL valves) transcription factors following FAL. (C-E) Loss of perivalvular endothelial expression of the antithrombotic proteins THBD ( $n=$ 9 control, FAL valves), EPCR ( $n=9$ control, $n=8$ FAL valves), and TFPI ( $n=7$ control, $n=9$ FAL valves) following FAL. (F) Gain of perivalvular endothelial expression of the prothrombotic protein vWF following FAL ( $n=6$ control, $n=8$ FAL valves). White dashed lines indicate luminal venous endothelial cells, and green dotted lines indicate perivalvular endothelial cells. For all graphs the mean is represented by the bar with each dot representing a replicate, and error bars indicate SD. Significance was determined by a 2-tailed Mann-Whitney test. ${ }^{*} P<0.05$. saccharide-treated Foxc2 ${ }^{\text {VVKO }}$ animals were observed in the deep venous system (at the anastomosis of the inferior vena cava and the lumbar veins), the site of clinically significant human venous thrombosis. These functional studies support a mechanism in which localized endothelial expression of the FOXC2 transcription factor supports a strong antithrombotic phenotype in the venous perivalvular area.

The antithrombotic venous perivalvular endothelial phenotype is conferred by hemodynamic forces. Since hemodynamic forces and OSS are known to drive the expression of the FOXC2, GATA2, and PROX1 transcription factors in perivalvular endothelial cells during valve formation (12-17), the studies described above suggested that OSS might be required for the antithrombotic phenotype displayed by perivalvular venous endothelial cells. To test an association between blood flow, expression of FOXC2 and PROX1, and the antithrombotic endothelial phenotype around the venous valve, we performed femoral artery ligation (FAL) to reduce the level of blood flow in the vein without directly manipulating the vein itself $(30,31)$. FAL resulted in an approximately $50 \%$ reduction in venous flow assessed at 72 hours by direct visualization of injected fluorescent beads and laser Doppler imaging (Supplemental Figure 7 and Supplemental Video 1). Immunostaining performed 72 hours after FAL revealed reduced expression of FOXC2 and PROX1 in perivalvular endothelial cells (Figure 5, A and B). Expression of the antithrombotic proteins THBD, EPCR, and TFPI also dropped significantly in perivalvular endothelial cells (Figure 5, C-E). Notably, expression of the prothrombotic protein vWF rose in perivalvular venous endothelial cells 72 hours after FAL (Figure 5F), demonstrating coordinate regulation of prothrombotic and antithrombotic gene expression and suggesting that the reduced expression of transcription factors and antithrombotic proteins is not merely reflective of globally reduced gene expression. The expression of ICAM1 in the perivalvular endothelium was not significantly altered 72 hours after FAL (Supplemental Figure 4C). These studies further link expression of FOXC2 and PROX1 to blood flow in vivo and support the conclusion that the local antithrombotic phenotype of venous perivalvular endothelium is conferred by specific hemodynamic conditions.

Muscular activity stimulates oscillatory blood flow in the venous valve sinus. Immobility is a well-defined risk factor for DVT that has been proposed to be linked to changes in venous blood flow (often called venous stasis), but deep venous blood flow remains high in the resting state owing to pumping of the heart and basal cardiac output. To determine whether immobility might influence the risk of DVT through effects on specific hemodynamic factors such as OSS that are linked to the transcriptional mechanisms described above, we examined blood flow in the veins of the human leg under conscious, immobile conditions, and immediately follow- 
A
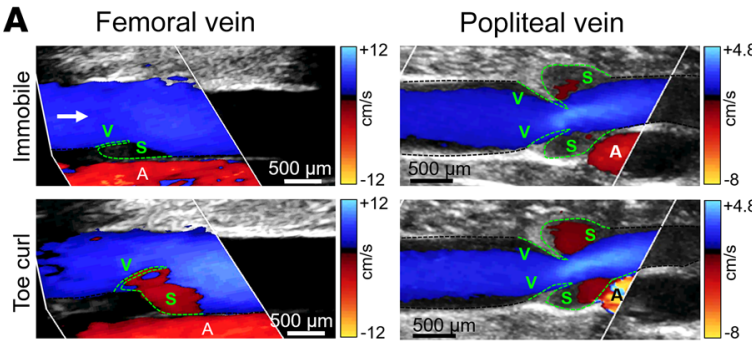

B

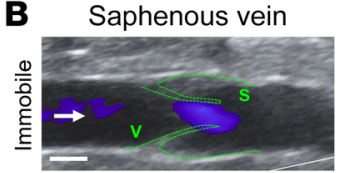

Femoral vein

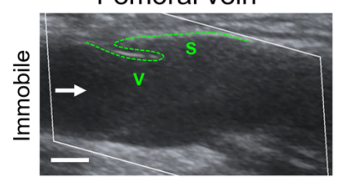

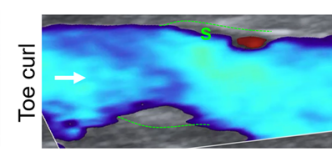

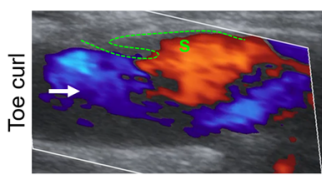

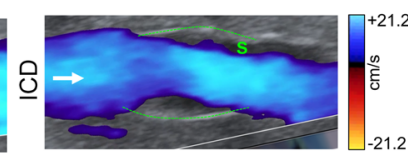

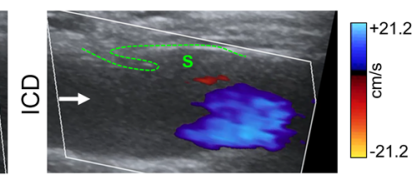

$\left\lfloor-\mathrm{C}^{2}\right.$
Saphenous vein
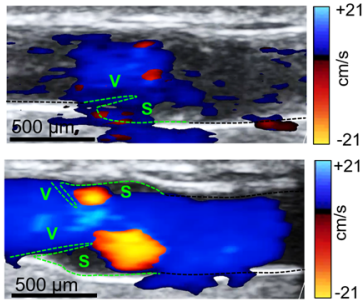

Figure 6. Oscillatory flow in the venous valve sinus is stimulated by muscular activity but not pneumatic compression of the calf. (A) $2 \mathrm{D}$ color Doppler studies of venous flow at the site of valves ( $V$, green dashed line) and valve sinuses $(S)$ in human femoral, popliteal, and saphenous veins were performed in healthy individuals. The images are representative of 3 femoral veins, 7 popliteal veins, and 7 saphenous veins studied in distinct individuals. Blood flow in the direction of circulation is shown in blue, and reverse/oscillatory flow in red and orange. Flow measured when the individual was immobile (top) was compared with flow measured during muscular activity stimulated by a single toe curl (bottom). A, adjacent artery. (B) Representative images of valve sinus (S) flow in healthy individuals in the saphenous and femoral veins during immobility, after a toe curl, and during the compression phase of a calf intermittent compression device (ICD). The images are representative of 3 saphenous veins and 4 femoral veins. ing muscular activity in the foot (a single toe curl). Color Doppler ultrasound studies revealed that the leg veins of immobile individuals experience forward flow in the lumen, with little flow detected in the valve sinus (Figure 6, A and B, and Supplemental Video 2). However, muscular activity stimulated strong forward flow in the lumen and reversing or oscillatory flow in the valve sinus (S) of the femoral, popliteal, and saphenous veins (Figure 6, A and B, and Supplemental Video 2). Prevention of DVT is presently approached mechanically through the use of intermittent compression devices (ICDs) that compress parts of the foot and leg to increase venous return (32-34). Numerous ICDs have been reported to increase the centerline velocity of venous flow $(32,34)$, but whether they also stimulate perivalvular oscillatory flow and OSS is not known. Doppler ultrasound analysis of the saphenous and femoral veins of healthy volunteers wearing a calf compression device currently used at the Hospital of the University of Pennsylvania (Flowtron Universal) revealed increased centerline luminal venous blood flow during ICD compression, but, unlike muscular activity, minimal oscillatory flow was observed in the valve sinus (Figure 6B and Supplemental Video 3). These findings reveal that oscillatory flow around the venous valve is primarily stimulated by muscular activity and suggest that existing ICDs may be relatively ineffective in stimulating perivalvular oscillatory flow.

Human DVT is associated with loss of the venous perivalvular endothelial transcription factor and antithrombotic phenotypes. The studies described above support a model in which OSS stimulated by muscular activity normally prevents DVT formation by maintaining local endothelial expression of the FOXC2 and PROX1 transcription factors that in turn confer a powerful antithrombotic phenotype in perivalvular endothelial cells (Figure 7, A and B). Such a model predicts that DVT in human patients would be associated with a loss of these unique perivalvular venous endothelial phenotypes, as observed in mice following FAL or genetic loss of FOXC2 or PROX1. To test this prediction, we examined the femoral veins of a 74-year-old individual who died suddenly of DVT and associated pulmonary embolism. At autopsy a large DVT was observed originating from the valve at the junction of the right superficial and deep femoral veins (Figure 7C, bottom), while the equivalent valve in the left leg did not have an associated DVT (Figure 7C, top). Histologic analysis revealed high FOXC2, PROX1, THBD, EPCR, and TFPI expression and low vWF expression in the endothelial cells lining the unaffected left femoral venous valve sinus and leaflet (Figure 7, D-J, top), a pattern identical to that observed in the healthy human saphenous vein (Supplemental Figure 2). In contrast, the endothelial cells lining the right femoral venous valve sinus and leaflet exhibited loss of FOXC2, PROX1, THBD, EPCR, and TFPI expression and gain of vWF expression (Figure 7, D-J, bottom). Identical molecular findings were observed in a second individual who also died of DVT and fatal pulmonary embolism (Supplemental Figure 8). The individuals studied had been in the hospital for multiple days prior to their venous thromboembolic events, consistent with a history of immobility and known risk for DVT. While it cannot be known for certain whether and to what extent the observed molecular changes preceded DVT formation in these individuals, these findings are consistent with a causal role for loss of the perivalvular endothelial transcription factor and antithrombotic phenotype in the pathogenesis of human DVT and venous thromboembolism.

\section{Discussion}

The concept that venous stasis associated with physical immobility is a major factor in DVT pathogenesis has been appreciated for over 150 years, but a mechanistic basis for this observation has not been established. Our studies demonstrate that hemodynamic forces generated around venous valves by muscular activity maintain expression of the hemodynamically regulated transcription factors FOXC2 and PROX1 that confer a powerful antithrombotic endothelial phenotype in the valve sinus, the known site of DVT origin. In mice reduced venous flow or loss of FOXC2 is sufficient to reverse this local antithrombotic phenotype and predispose toward perivalvular venous thrombosis. Consistent with these experimental observations in mice, the perivalvular endothelium at the site of origin of human DVT exhibits identical loss of this unique transcription factor and antithrombotic phenotype, and human physi- 
A Normal venous flow

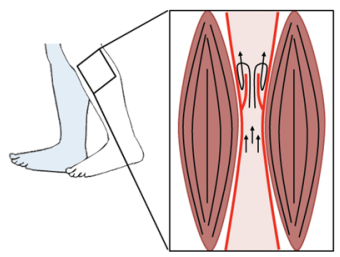

Perivalvular oscillatory flow $(\hat{j}$

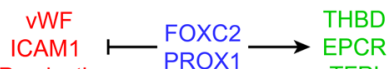

$\underset{\text { P-selectin }}{\mathrm{ICAM} 1} \underset{\mathrm{PROX} 1}{\mathrm{FOXC2}} \underset{\mathrm{TFPI}}{\mathrm{EPCR}}$
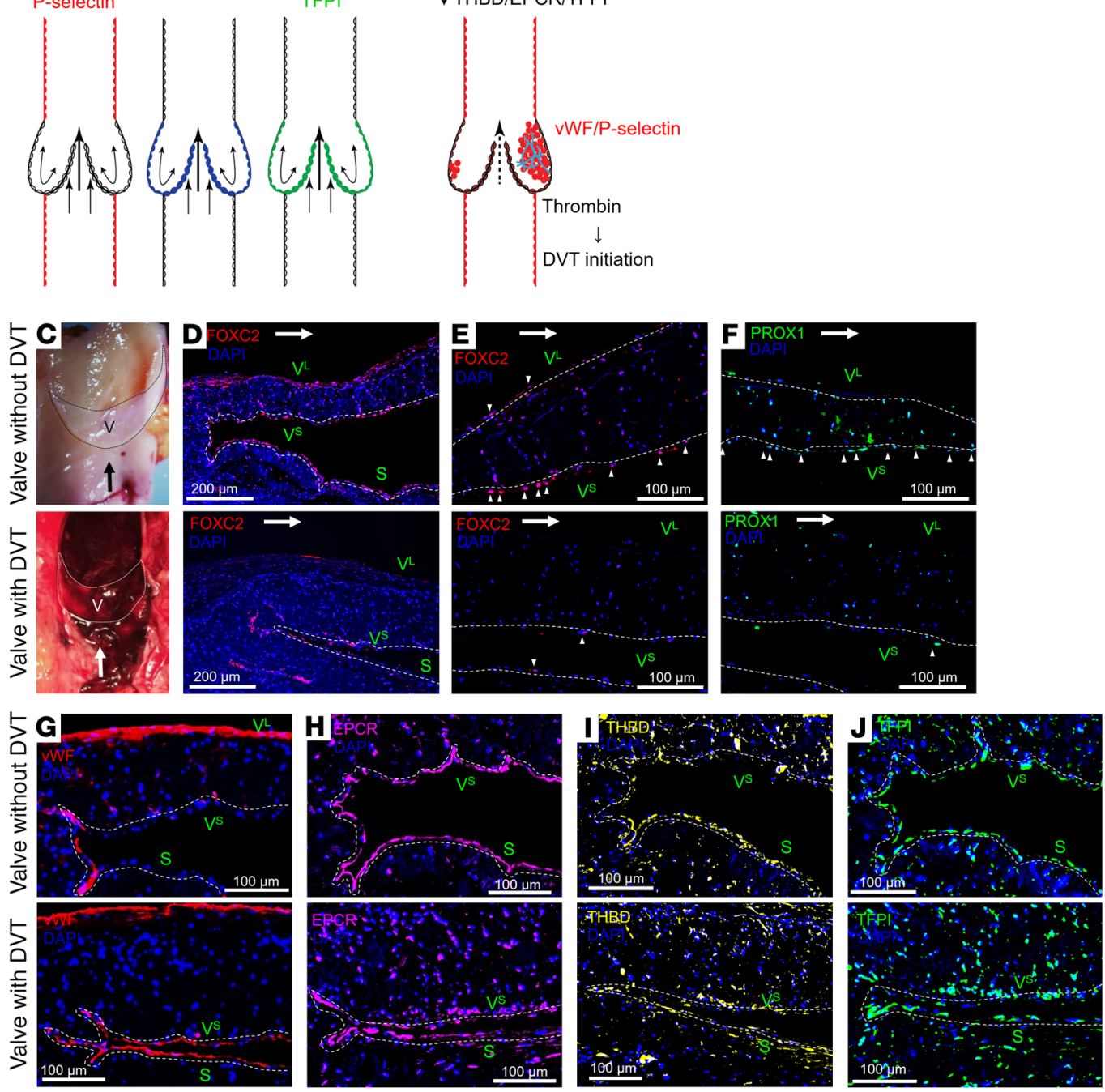

Figure 7. Human DVT arises in association with loss of the perivalvular endothelial transcription factor and antithrombotic phenotypes. (A and B)

Model of the relationship between muscular activity, perivalvular oscillatory flow, perivalvular endothelial cell gene expression, and venous thrombosis. In the active state, muscular activity stimulates oscillatory flow in the venous valve sinus, driving endothelial expression of the shear-regulated FOXC2 and PROX1 transcription factors. Shear-regulated transcription factor activity upregulates expression of the antithrombotic proteins THBD, EPCR, and TFPI while simultaneously downregulating expression of the prothrombotic proteins vWF, P-selectin, and ICAM1 to maintain a highly antithrombotic environment (A). Inactivity and lack of muscular contraction results in perivalvular endothelial loss of expression of the shear-regulated transcription factors, loss of THBD, EPCR, and TFPI expression, and gain of VWF, P-selectin, and ICAM1 expression, resulting in thrombin generation and formation of DVT (B). (C-E) Human DVT is associated with loss of endothelial expression of FOXC2 and PROX1 in the valve sinus. (C) After death due to pulmonary embolism, femoral vein samples containing the valve at the anastomosis of the superficial and deep femoral veins were harvested from a leg without DVT (top) and from the contralateral leg with DVT in situ (bottom). (D-F) Immunostaining for FOXC2 and PROX1 were performed on sections from the valves shown in C. Arrowheads indicate FOXC2 ${ }^{+}$and PROX1 ${ }^{+}$nuclei in $\mathbf{E}$ and $\mathbf{F}$. (G-J) DVT is associated with endothelial gain of expression of the prothrombotic protein vWF and loss of the antithrombotic proteins THBD, EPCR, and TFPI in the valve sinus. The white dashed line indicates the endothelial layer. $\mathrm{V}^{\mathrm{L}}$, luminal face of the valve; $\mathrm{V}^{5}$, sinus face of the valve; $\mathrm{S}$, vessel wall in the valve sinus. 
ologic studies directly connect muscular activity to oscillatory flow at the venous valve. Together these studies provide a hemodynamic, cellular, and molecular mechanism for DVT that explains its established association with immobility and has immediate implications for the treatment of this common and lethal disease.

The first mechanism for DVT proposed by Virchow was "venous stasis" (3), and the best established clinical risk factor for DVT is immobility (35). Venous stasis has come to be defined as changes in venous blood flow that are associated with physical immobility, but why immobility should confer important changes in venous blood flow is not immediately apparent, because blood flow in the large veins of the leg (where DVTs arise) is determined primarily by cardiac output and therefore remains high even in an immobile individual. A clue to the mechanism by which mobility might alter DVT risk emerged in the 1960s and 1970s when autopsy studies revealed that even very large DVTs arise from small thrombi that form in the venous valve sinus (7-9). Venography of immobile individuals revealed slow exchange of blood in the valve sinus compared with the rest of the vein (10), suggesting that the valve sinus might be a specific site of hemodynamic stasis that triggers clot formation and DVT. However, more recent studies revealed an antithrombotic phenotype among the endothelial cells lining the venous valve sinus $(23,36)$, suggesting that this region should be at lower risk for clot formation than the rest of the vein, and raising the question of why DVTs would form preferentially at that site. Our studies resolve these important observations and support a mechanism in which muscular activity associated with physical mobility generates oscillatory or reversing blood flow in the venous valve sinus that upregulates expression of the FOXC2 and PROX1 transcription factors, known to be activated by OSS, which in turn maintain a strong antithrombotic and antiinflammatory environment around the venous valve (Figure 7A). We observe significant loss of this local transcription factor and antithrombotic phenotype only 72 hours after reducing venous blood flow in mice, suggesting that this antithrombotic phenotype is highly dynamic and explaining how even relatively short periods of immobility (such as long plane flights) may significantly raise the risk of DVT formation.

Virchow's second proposed mechanism for DVT pathogenesis was vessel wall injury (3). Since histologic studies have failed to demonstrate physical injury at the site of DVT formation, this mechanism has more recently come to be interpreted as vessel wall inflammation $(6,37)$, but a molecular and/or cellular basis for such local inflammation has not been identified. Our studies do not demonstrate a true inflammatory endothelial phenotype around the venous valve following loss of venous blood flow or endothelial expression of FOXC2 or PROX1, but they do reveal changes in surface P-selectin expression that support leukocyte rolling and adhesion at that site. A role for leukocytes in thrombus formation is well established, and particularly important in venous thrombosis $(8,24-26)$. Leukocytes express tissue factor required to activate coagulation proteases and thrombin generation, as well as neutrophil extracellular traps that physically support clot formation (reviewed in ref. 38). Venous thrombi form at much lower shear forces than arterial thrombi such as those responsible for myocardial infarction and stroke, and incorporate large numbers of circulating white and red blood cells in addition to platelets.
Thus local vascular "inflammation" implicated in DVT formation may also reflect perivalvular endothelial gene expression changes connected to the loss of OSS and the hemodynamically stimulated FOXC2 and PROX1 transcription factors as a consequence of immobility. When these local factors are combined with a systemic hypercoagulable state (Virchow's third mechanism), the net effect is formation of DVT at the site of the venous valve.

Our studies and those previously reported $(23,36)$ reveal a previously unrecognized hemodynamic and transcriptional mechanism by which intravascular thrombosis is spatially regulated in vivo. Thrombin is the key regulator of clot formation in vivo because it both activates the cells involved in clot formation (e.g., platelets and leukocytes) and cleaves circulating fibrinogen to create cross-linked fibrin (reviewed in ref. 39). Coexpression of THBD and EPCR on the perivalvular endothelial cell surface is predicted to create a highly antithrombotic local environment, because thrombin bound to THBD efficiently activates protein $\mathrm{C}$ bound to EPCR, resulting in powerful negative feedback that ultimately stops thrombin generation and prevents clot formation $(40,41)$. When local activated protein $C$ generation is combined with local TFPI expression to block thrombin generation, the loss of $\mathrm{vWF}$ required for efficient platelet recruitment, and the loss of P-selectin and ICAM1 required for efficient leukocyte recruitment, the result is a powerful, synergistic molecular inhibition of thrombus formation at the venous valve (Figure 7A).

The discovery of a specific hemodynamic requirement in the venous valve sinus to prevent loss of the perivalvular antithrombotic phenotype has immediate implications for the clinical approaches to DVT among high-risk patients, such as those in hospital for surgery and trauma. Present treatment and prevention of DVT consists of systemic anticoagulation and pneumatic compression devices designed to augment venous flow in the legs. Since many patients at the highest risk for DVT are also at high risk for hemorrhage due to recent surgery or trauma, systemic anticoagulation is often not an option. Pneumatic compression devices are widely used to reduce the incidence of DVT in hospitalized patients (42-44), but these devices have been designed and applied without a clear understanding of how changing venous hemodynamics might protect against DVT, and we observe only weak stimulation of perivalvular oscillatory flow with one such commonly used device. Thus it is likely that mechanical therapy could be significantly improved if it were targeted more specifically to reestablish the oscillatory flow in the venous valve sinus normally driven by muscular activity. Consistent with this notion, it has been appreciated for over 50 years that devices that provide electrical stimulation to the leg muscles during operative procedures - an approach predicted to more closely reproduce the hemodynamic effects of normal muscular activity - are highly effective in preventing DVT (although they are not well tolerated by conscious individuals) (45-47). Thus the creation of new mechanical therapies designed to specifically restore oscillatory flow in the venous valve sinus is predicted to improve the prevention of DVT in large numbers of high-risk individuals.

\section{Methods}

Human studies. The superficial saphenous, popliteal, and femoral veins were analyzed by ultrasound for flow direction and velocity at the site of venous valves. Subjects were between the ages of 25 and 
55 with no history of venous disease, and were an even mix of female and male. The veins were examined in transverse section to identify venous valves by visualizing the valve leaflet and turbulent flow in the sinus with duplex ultrasonography. Venous flow was measured using 2D color Doppler with the volunteer prone either not moving, during a single toe curl, or during the compression cycle of an ICD device (Flowtron Universal calf compression pump, Huntleigh). The ultrasounds were performed with either a Mindray M9 portable ultrasound machine with an L12-4s linear array ultrasound transducer, or a Phillips EPIC 7G with an L12-3 transducer.

Mouse studies. All experimental animals were maintained on a mixed 129/SvJ, C57BL/6J, DBA/2J genetic background and littermate controls used unless specifically described. PROX1-GFP BAC transgenic mice (48) were obtained from Young-Kwon Hong at the University of Southern California, Los Angeles, California. Prox1 $1^{\text {CreERT2 }}$ mice were obtained from The Jackson Laboratory. Foxc $2^{f l}$ mice (49) were a gift from Tsutomu Kume (Northwestern University, Chicago, Illinois, USA), and GATA2-GFP reporter mice (50) were a gift from James Engel (University of Michigan Medical School, Ann Arbor, Michigan, USA). $\operatorname{Prox} 1^{f l f l} C d h 5^{\text {CreERT2 }}$ mice were generated in the laboratory of Guillermo Oliver (Northwestern University). All adult Cre $\mathrm{CRT2}^{\text {ERT }}$ deletions (both Prox1-CreERT2 and Cdh5-CreERT2) were induced by oral gavage of $200 \mu \mathrm{L}$ of $0.025 \mathrm{~g} / \mathrm{mL}$ tamoxifen (Sigma-Aldrich) for 5 straight days followed by maintenance on tamoxifen chow (Harlan/Envigo) until the time of venous harvest.

Defining the venous valve sinus region. The valve sinus is defined, in humans or in mice without PROX1-GFP expression, as the vessel wall endothelium that is downstream of the venous valve leaflets between the sites where the valve leaflet joins the vessel wall (Supplemental Figure 9, A and B). Perivalvular endothelium refers to the endothelium within the sinus region including both the valve leaflet and wall endothelium within this region. This site is consistently the only site of high venous expression of PROX1, as indicated by PROX1-GFP mice (Supplemental Figure 9C), which can also be used to define the valve sinus. When applicable, we used PROX1-GFP or PROX1 antibody staining to define the valve sinus in both intravital imaging and histologic sections. When those markers were not available we used the anatomic definition of the sinus endothelium to demarcate the region.

Human and mouse venous valve isolation. Venous valves were collected from adult mice (8-16 weeks old) of both sexes by removing the portion of the superficial saphenous vein and femoral vein at the sapheno-femoral junction where a stereotypical valve is present. The presence of this valve was consistent across mouse sexes and strains. Venous valves from humans were obtained from the saphenous vein remnants from patients undergoing artery bypass grafting. Femoral vein samples were obtained from autopsies of patients who died of pulmonary embolism where the clot was visible in the vessel. All vessels were washed in sterile saline and fixed in $4 \%$ paraformaldehyde (Electron Microscopy Sciences) overnight, and then dehydrated in ethanol. The vessels were embedded longitudinally in paraffin and cut to $6-\mu \mathrm{m}$-thick sections for fluorescent immunohistochemical staining.

Immunohistochemistry and antibodies. All tissue was fixed in $4 \%$ paraformaldehyde overnight and then dehydrated stepwise in ethanol (EtOH) as follows: $0 \% \mathrm{EtOH}$ in PBS (2 hours at $\left.4^{\circ} \mathrm{C}\right), 50 \% \mathrm{EtOH}$ (2 hours at $4^{\circ} \mathrm{C}$ ), $70 \% \mathrm{EtOH}$ (overnight at $4^{\circ} \mathrm{C}$ ); and finally stored in $100 \% \mathrm{EtOH}$ at $-20^{\circ} \mathrm{C}$. The tissue was embedded in paraffin and cut into $6-\mu \mathrm{m}$-thick sections for staining.
Primary antibodies used for immunostaining were as follows: anti-vWF (human and mouse, Dako), anti-human thrombomodulin (clone 141C01, Thermo Fisher Scientific), anti-human EPCR (clone LMR-42, Invitrogen), anti-human GATA2 (polyclonal, Santa Cruz Biotechnology), anti-human FOXC2 (clone 665819, R\&D Systems), anti-mouse thrombomodulin (clone 461714, Thermo Fisher Scientific), anti-mouse PROX1 (clone 76696, Abcam), anti-mouse EPCR (catalog 60038, STEMCELL Technologies), anti-GFP (catalog AB6673, Abcam), anti-mouse TFPI (polyclonal, Abcam), anti-mouse ICAM1 (catalog 14-0541, eBioscience), and anti-mouse CD62P (clone RB40.34, BD Pharmingen).

Microscopy. Fluorescent immunohistochemical data were imaged with a Nikon Eclipse 80i epifluorescence microscope $(\times 10$ or $\times 20$ dry objective). Intravital imaging of clot formation was done using an Olympus MVX10 dissecting scope fitted with an Olympus DP74 color camera and an Xcite 120 LED Boost light source (Olympus). Intravital imaging of P-selectin surface expression was done using an Olympus BX61WI upright microscope with a $\times 10$ water immersion objective after infusion of $4 \mu \mathrm{g}$ anti-P-selectin (clone RB40.34, BD Biosciences) labeled with Alexa Fluor 647 (Invitrogen).

Intravascular thrombosis assay. Adult mice (8-16 weeks) were anesthetized with $1 \%-3 \%$ isoflurane and maintained on a $37^{\circ} \mathrm{C}$ heating pad. Retro-orbital injection of $100 \mu \mathrm{L}$ of rhodamine 6G $(1 \mathrm{mM})$ was used to label platelets and white blood cells, and fibrin was labeled with anti-fibrin antibody (clone 59D8; a gift from Tim Stalker and Rodney Camire) labeled with Alexa Fluor 488 using the Alexa Fluor Monoclonal Antibody labeling kit, according to the manufacturer's instructions (Invitrogen). The mouse saphenous vein was surgically exposed at the site of saphenous/epigastric vein confluence where a stereotypic venous valve is found. To induce thrombus formation on intact endothelium, the vessel was cleared of connective tissue, avoiding damage, and covered in a solution of $0.3 \mathrm{mg} / \mathrm{mL}$ of human $\alpha$-thrombin (Haematologic Technologies Inc.) in sterile PBS. At least $250 \mu \mathrm{m}$ of vessel was covered in thrombin solution both upstream and downstream from the valve. Intravital fluorescence imaging was used to monitor thrombus formation for either 2 or 10 minutes after thrombin application. The endothelial thrombogenicity was quantified by measurement of the percentage of the vessel containing a thrombus upstream and downstream from the valve/sinus area and within the valve/sinus. Thrombus formation was quantified as percentage of vessel coverage because the intravital imaging provides a top-down view of the vessel wall where the thrombi form, which prevents accurate measurement of total thrombus height and size. The valve and sinus areas were defined by endothelial PROX1-GFP expression.

Activated partial thromboplastin time. Blood was obtained from Foxc $2^{f / f l}$ and Foxc2 ${ }^{\text {VVKO }}$ littermates from inferior vena cava blood draws into 1:9 dilution of $3.8 \%$ sodium citrate. Platelet-poor plasma (PPP) was obtained through centrifugation at $3000 \mathrm{~g}$ for 10 minutes. PPP was diluted with activated partial thromboplastin time (APTT) reagent (Pacific Hemostasis APTT kit, Thermo Fisher Scientific) using Ellagic Acid Activator to induce clotting, and time to full clot formation reported.

Fibrin clot formation. PPP was obtained from Foxc $2^{f / f l}$ and Foxc2 $2^{\text {VVKO }}$ littermates as described above. Briefly, PPP was recalcified with $\mathrm{CaCl}_{2}$ $(20 \mathrm{mM})$ in a well plate at $37^{\circ} \mathrm{C}$, and $405-\mathrm{nm}$ absorbance readings were recorded once per minute for an hour to track fibrin deposition. The $t_{1 / 2}$ time of fibrin clot formation was calculated and reported. 
Leukocyte rolling. Mice were prepared for surgery as described above and infused with rhodamine $6 \mathrm{G}$, and the saphenous vein was exposed to allow for visualization of the saphenous vein valves. Leukocyte rolling was visualized using intravital microscopy, and quantified by measurement of the number of leukocytes rolling per millimeter of vessel upstream and downstream from the valve/sinus area and within the valve/sinus. Intraperitoneal injection of $2.5 \mathrm{EU} / \mathrm{kg}$ of lipopolysaccharide 4 hours before imaging was used to increase endothelial cell activation and increase leukocyte rolling.

Femoral artery ligation. Mouse femoral artery ligation (FAL) and laser Doppler imaging procedures were done as previously described (31). Briefly, mice were prepared for surgery as described above, and bupivacaine and meloxicam were administered to the mice for pain management. After surgical exposure of the femoral artery of the mice, a single occluding suture was tied around the femoral artery proximally to the femoral artery bifurcation. The mice were removed from anesthesia and allowed to recover. Blood perfusion of the ligated and nonligated legs was measured by laser Doppler imaging at 24 hours and 72 hours after surgery. At 72 hours, $100 \mu \mathrm{L}$ of FluoSpheres (1- $\mu$ m beads, 580/605, Invitrogen) were infused to measure blood flow velocity in saphenous vein in both the ligated and the nonligated legs of the mouse.

Quantification and statistics. All fluorescent images were analyzed and quantitated using Image (NIH). Because of the technical challenges of histologic analysis of mouse saphenous vein valves, it was not possible to consistently get sections of venous valves from different mice on the same slide for immunohistochemical staining. To control for slide-to-slide variation in fluorescent staining, we internally controlled the fluorescent values of the venous valve and sinus endothelium relative to the lumen endothelium (Supplemental Figure 10). Thus, the valve and sinus endothelium data are reported as fold change of their respective lumen endothelium. Fluorescence intensity was quantified per tissue area for all non-nuclear staining, and for nuclear-specific fluorescent signal (PROX1 and FOXC2) we normalized per nucleus. The background signal for each slide was measured and subtracted from the image before normalization.

In order to statistically evaluate the differences between these groups, we used the ratio paired $t$ test, which compares the ratio difference between each lumen-valve or lumen-sinus pair. In comparisons of the lumen endothelium with the valve and sinus endothelium, we used the Bonferroni correction to adjust the significance level for multiple comparisons, and so a $P$ value less than 0.025 was considered significant. To analyze statistical differences between valve and sinus endothelium between groups (such as Foxc $2^{f / f l}$ and $F o x c 2^{V V K O}$ ), we used the unpaired nonparametric Mann-Whitney test to compare the fold change values compared with their respective lumenal endothelium. In the Foxc $2^{\text {VVКо }}$, Prox $1^{\text {EКо }}$, and FAL experiments, single comparisons between the valve leaflet and sinus endothelium of the control group and the treatment groups were performed, so no correction was used, and a $P$ value less than 0.05 was considered significant. An unpaired, 2-tailed $t$ test was used to compare hemodynamic conditions in the femoral ligation model and peak flow velocity of human vein ultrasound data, and a $P$ value less than 0.05 was considered significant.
The extent of leukocyte rolling within the valve/sinus, and the extent of thrombus formation in the intravascular thrombosis model, compared the valve/sinus region with both the upstream and downstream vessel portions, so again the Bonferroni correction was used and a $P$ value less than 0.025 was considered significant. To compare the rates of spontaneous microthrombus formation in Foxc $2^{f / f l}$ and Foxc2 $2^{V V K O}$ mice, we used a 2-sided Fisher's exact test, as this was a binary readout, and a $P$ value less than 0.05 was considered significant.

Study approval. The University of Pennsylvania Institutional Review Board approved all human protocols, and all procedures were performed in accordance with these protocols, including informed consent from all subjects. The University of Pennsylvania Institutional Animal Care and Use Committee approved all animal protocols, and all procedures were performed in accordance with these protocols.

\section{Author contributions}

JDW designed and performed most of the experiments and helped write the manuscript. MHH and SS performed the human Doppler studies. SB assisted JDW in numerous experimental studies. JDH and JMJ designed and performed studies examining venous endothelial responses to fluid flow. JY performed histo-

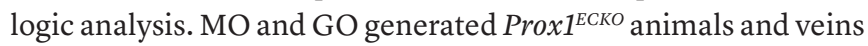
for study. LL designed and performed experiments. JDE provided GATA2-GFP reporter animals. TK provided critical reagents. JDW, MHH, and MLK helped design and perform the experiments and wrote the manuscript.

\section{Acknowledgments}

We thank members of the Kahn laboratory for their thoughtful comments during the course of this work. We thank Carrie Sims and Ben Abella and the Penn Acute Research Collaborative for assistance in obtaining human saphenous vein samples. We thank Rodney Camire from the Children's Hospital of Philadelphia for assistance in performing the APTT assay. We thank Chandra Sehgal for assistance in examining venous blood flow using Doppler ultrasound. We thank Joseph A. DiRienzi, Igor Tsimberg, and Leslie A. Litzky of the Section for Medical Pathology at the University of Pennsylvania for invaluable assistance in obtaining human venous DVT samples at autopsy. We also thank Wanshu Ma and Xiaolei Liu from the Oliver laboratory for help with the generation of animal samples. These studies were supported by NIH grants R01HL121650 (to MLK), P01HL120846 (to MLK), R01HL120872 (to MLK), T32HL07439 (to JDW), RO1HL126920 (to TK), and RO1HL073402 (to GO).

Address correspondence to: Mark L. Kahn, 11-123 Smilow Center for Translational Research, 3400 Civic Center Boulevard, Building 421, Philadelphia, Pennsylvania 19104-5159, USA. Phone: 215.898.9007; Email: markkahn@mail.med.upenn.edu.

MH's present address is: Department of Surgery, Section of Acute and Critical Care Surgery, Washington University in St. Louis, St. Louis, Missouri, USA.
1. Cushman M. Epidemiology and risk factors for venous thrombosis. Semin Hematol. 2007;44(2):62-69.

2. Beckman MG, Hooper WC, Critchley SE, Ortel TL.
Venous thromboembolism: a public health concern. Am JPrev Med. 2010;38(4 suppl):S495-S501.

3. Virchow R. Gesammelte Abhandlungen zur wissenschaftlichen Medicin. Frankfurt, Germany:
Meidinger Sohn; 1856.

4. Alhenc-Gelos M, Gandrille S, Aubry ML, Emmerich J, Flessinger JN, Aiach M. Unexplained thrombosis and factor V Leiden mutation. Lan- 
cet. 1994;344(8921):555-556.

5. Baker R, Thom J, van Bockxmeer F. Diagnosis of activated protein $\mathrm{C}$ resistance (factor $\mathrm{V}$ Leiden). Lancet. 1994;344(8930):1162.

6. Hisada Y, Geddings JE, Ay C, Mackman N. Venous thrombosis and cancer: from mouse models to clinical trials. J Thromb Haemost. 2015;13(8):1372-1382

7. Paterson JC, McLachlin J. Precipitating factors in venous thrombosis. Surg Gynecol Obstet. 1954;98(1):96-102.

8. Sevitt S. The structure and growth of valvepocket thrombi in femoral veins. J Clin Pathol. 1974;27(7):517-528.

9. Sevitt S. Organization of valve pocket thrombi and the anomalies of double thrombi and valve cusp involvement. Br J Surg. 1974;61(8):641-649.

10. McLachlin AD, McLachlin JA, Jory TA, Rawling EG. Venous stasis in the lower extremities. Ann Surg. 1960;152:678-685.

11. Petrova TV, et al. Defective valves and abnormal mural cell recruitment underlie lymphatic vascular failure in lymphedema distichiasis. Nat Med. 2004;10(9):974-981.

12. Sabine A, et al. Mechanotransduction, PROX1, and FOXC2 cooperate to control connexin 37 and calcineurin during lymphatic-valve formation. Dev Cell. 2012;22(2):430-445.

13. Sweet DT, et al. Lymph flow regulates collecting lymphatic vessel maturation in vivo. JClin Invest. 2015;125(8):2995-3007.

14. Cha B, et al. Mechanotransduction activates canonical Wnt/ $\beta$-catenin signaling to promote lymphatic vascular patterning and the development of lymphatic and lymphovenous valves. Genes Dev. 2016;30(12):1454-1469.

15. Srinivasan RS, Oliver G. Prox1 dosage controls the number of lymphatic endothelial cell progenitors and the formation of the lymphovenous valves. Genes Dev. 2011;25(20):2187-2197.

16. Kazenwadel J, et al. GATA2 is required for lymphatic vessel valve development and maintenance. J Clin Invest. 2015;125(8):2979-2994.

17. Kume T. Lymphatic vessel development: fluid flow and valve-forming cells. J Clin Invest. 2015;125(8):2924-2926.

18. Sabine A, et al. FOXC2 and fluid shear stress stabilize postnatal lymphatic vasculature. JClin Invest. 2015;125(10):3861-3877.

19. Mellor RH, et al. Mutations in FOXC2 are strongly associated with primary valve failure in veins of the lower limb. Circulation. 2007;115(14):1912-1920.

20. Surendran S, et al. Forkhead box C2 promoter variant c. $-512 \mathrm{C}>\mathrm{T}$ is associated with increased susceptibility to chronic venous diseases. PLoS One. 2014;9(3):e90682.

21. Lyons O, et al. Human venous valve disease caused by mutations in FOXC2 and GJC2. J Exp
Med. 2017;214(8):2437.

22. Bazigou E, et al. Genes regulating lymphangiogenesis control venous valve formation and maintenance in mice. J Clin Invest. 2011;121(8):2984-2992.

23. Brooks EG, et al. Valves of the deep venous system: an overlooked risk factor. Blood. 2009;114(6):1276-1279.

24. von Brühl ML, et al. Monocytes, neutrophils, and platelets cooperate to initiate and propagate venous thrombosis in mice in vivo. JExp Med. 2012;209(4):819-835.

25. Myers D, et al. Selectins influence thrombosis in a mouse model of experimental deep venous thrombosis. J Surg Res. 2002;108(2):212-221.

26. Celi A, et al. Thrombus formation: direct realtime observation and digital analysis of thrombus assembly in a living mouse by confocal and widefield intravital microscopy. J Thromb Haemost. 2003;1(1):60-68.

27. Denis CV, André P, Saffaripour S, Wagner DD. Defect in regulated secretion of P-selectin affects leukocyte recruitment in von Willebrand factor-deficient mice. Proc Natl Acad Sci U S A. 2001;98(7):4072-4077.

28. Choi EY, et al. Del-1, an endogenous leukocyte-endothelial adhesion inhibitor, limits inflammatory cell recruitment. Science. 2008;322(5904):1101-1104.

29. Liu Z, et al. Differential regulation of human and murine P-selectin expression and function in vivo. J Exp Med. 2010;207(13):2975-2987.

30. Niiyama H, Huang NF, Rollins MD, Cooke JP. Murine model of hindlimb ischemia. J Vis Exp. 2009;(23):1035.

31. Limbourg A, Korff T, Napp LC, Schaper W, Drexler H, Limbourg FP. Evaluation of postnatal arteriogenesis and angiogenesis in a mouse model of hind-limb ischemia. Nat Protoc. 2009;4(12):1737-1746.

32. Delis KT, Slimani G, Hafez HM, Nicolaides AN. Enhancing venous outflow in the lower limb with intermittent pneumatic compression. A comparative haemodynamic analysis on the effect of foot vs. calf vs. foot and calf compression. Eur J Vasc Endovasc Surg. 2000;19(3):250-260.

33. Whitelaw GP, Oladipo OJ, Shah BP, DeMuth KA, Coffman J, Segal D. Evaluation of intermittent pneumatic compression devices. Orthopedics. 2001;24(3):257-261

34. Morris RJ, Woodcock JP. Evidence-based compression: prevention of stasis and deep vein thrombosis. Ann Surg. 2004;239(2):162-171.

35. Engbers MJ, Blom JW, Cushman M, Rosendaal FR, van Hylckama Vlieg A. The contribution of immobility risk factors to the incidence of venous thrombosis in an older population. J Thromb Haemost. 2014;12(3):290-296
36. Trotman WE, Taatjes DJ, Callas PW, Bovill EG. The endothelial microenvironment in the venous valvular sinus: thromboresistance trends and inter-individual variation. Histochem Cell Biol. 2011;135(2):141-152.

37. Mackman N. New insights into the mechanisms of venous thrombosis. J Clin Invest. 2012;122(7):2331-2336.

38. Wolberg AS, et al. Venous thrombosis. Nat Rev Dis Primers. 2015;1:15006.

39. Coughlin SR. Thrombin signalling and protease-activated receptors. Nature. 2000;407(6801):258-264.

40. Esmon CT, Esmon NL, Harris KW. Complex formation between thrombin and thrombomodulin inhibits both thrombin-catalyzed fibrin formation and factor V activation. J Biol Chem 1982;257(14):7944-7947.

41. Healy AM, Rayburn HB, Rosenberg RD, Weiler $\mathrm{H}$. Absence of the blood-clotting regulator thrombomodulin causes embryonic lethality in mice before development of a functional cardiovascular system. Proc Natl Acad Sci U S A. 1995;92(3):850-854.

42. Calnan JS, Pflug JJ, Mills CJ. Pneumatic intermittent-compression legging simulating calf-muscle pump. Lancet. 1970;2(7671):502-503.

43. Hills NH, Pflug JJ, Jeyasingh K, Boardman L, Calnan JS. Prevention of deep vein thrombosis by intermittent pneumatic compression of calf. $\mathrm{Br}$ Med J. 1972;1(5793):131-135.

44. Ibrahim M, Ahmed A, Mohamed WY, El-Sayed Abu Abduo S. Effect of compression devices on preventing deep vein thrombosis among adult trauma patients: a systematic review. Dimens Crit Care Nurs. 2015;34(5):289-300.

45. Tichy VL. Prevention of venous thrombosis and pulmonary embolism by electrical stimulation of leg muscles. Surgery. 1949;26(1):109-116.

46. Doran FS, White HM. A demonstration that the risk of postoperative deep venous thrombosis is reduced by stimulating the calf muscles electrically during the operation. Br J Surg. 1967;54(8):686-689.

47. Browse NL, Negus D. Prevention of postoperative leg vein thrombosis by electrical muscle stimulation. An evaluation with 125I-labelled fibrinogen. Br Med J. 1970;3(5723):615-618.

48. Choi I, et al. Visualization of lymphatic vessels by Prox1-promoter directed GFP reporter in a bacterial artificial chromosome-based transgenic mouse. Blood. 2011;117(1):362-365.

49. Sasman A, et al. Generation of conditional alleles for Foxc1 and Foxc2 in mice. Genesis. 2012;50(10):766-774

50. Lim KC, et al. Conditional Gata2 inactivation results in HSC loss and lymphatic mispatterning. J Clin Invest. 2012;122(10):3705-3717. 